\title{
Análisis de la Dinámica del Mercado Agropecuario. Caso de una Asociación de Ganaderos
}

\author{
Dynamic Analysis of the Agricultural Market. \\ Case of an Association of Livestock Farmers \\ Briggette Estefanía VERA-SUÁREZ', Maribel Estefanía CRIOLLO-CENTENO', \\ Óscar Mauricio ROMERO-HIDALGO' (iD) y Martha Cecilia AGUIRRE-BENALCÁZAR' \\ ${ }^{1}$ Universidad Técnica de Machala, Facultad de Ciencias Empresariales. Machala, Ecuador.

\section{Resumen}

Esta investigación estuvo orientada hacia el estudio de mercado del sector ganadero. El objetivo principal fue analizar la dinámica del mercado agropecuario en la provincia de El Oro (Ecuador) por medio del caso de la Asociación de Ganaderos 24 de mayo.

Se indagó en fuentes de investigación científica utilizando el método descriptivo no experimental para estudiar las variables de mercado del sector. Además, se aplicó una encuesta a 159 personas de la localidad.

Respecto a los resultados, se comprobó que los productos ganaderos tienen alta demanda entre los consumidores, especialmente de las mujeres. Asimismo, se pudo observar que al ser productos de primera necesidad requieren de un incremento en su producción.

En conclusión, con base en una proyección entre los datos de la asociación y la población, se constató que la demanda de los clientes está insatisfecha. Esta situación se podrá minimizar si los productores implementan nuevos métodos para manufacturar los productos derivados del ganado, lo cual reportará múltiples beneficios.

Palabras clave

Ganadería, demanda, oferta, consumidores, mercado agropecuario.

\section{Abstract}

This research is oriented towards the market study of the livestock sector, whose main objective is to analyze the dynamics of the agricultural market in the province of El Oro, Ecuador, analyzing the case of the Association of Livestock Farmers May 24

For this, it has been investigated through different sources of scientific research, using the non-experimental descriptive method, in which the market variables of this sector are studied, strengthening it through a survey carried out on 159 people from the town. As a result, it was found that livestock products are in high demand by consumers, especially women; thus it could also be observed that since they are staple products, they require an increase in their production.

As a conclusion, it was observed that there is an unsatisfied demand from customers; establishing a projection between the data of the association and the population; This can be minimized if producers implement new methods for the manufacture of livestock products, thus reflecting multiple benefits.

\section{Keywords}

Livestock, demand, supply, consumers, agricultural market.

Códigos de clasificación JEL: L11, M30, Q11, Q13. 


\section{Introducción}

El sector agropecuario es uno de los más consistentes a nivel mundial. Su desarrollo data desde las primeras civilizaciones de la historia y, con el pasar del tiempo, ha ido tomando forma dentro de la economía de producción de plantas y animales en las sociedades europeas. Es importante destacar que la ganadería consiste en la explotación de animales para aprovechar al $100 \%$ su producción y derivados. Esta actividad es estudiada por la zootecnia la cual está relacionada con la agricultura. La agricultura, a su vez, fue la actividad que dio forma al surgimiento de los sectores agropecuarios.

El tiempo de reproducción del ganado en ciertos climas tropicales y subtropicales del mundo puede variar en un porcentaje del $45 \%$ al $55 \%$, con lapsos de 18 meses entre partos y superando los tres años en el caso de vacas primerizas. Los índices pueden deberse a factores genéticos (raza), problemas sanitarios factores o climáticos (humedad y radiación solar) (Verdolik et al., 2018). La reproducción de los bovinos depende por completo de su adaptación a los cambios de cada continente.

En este contexto, los productores europeos se enfrentan al conflicto de decidir si producir nuevas fuentes de empleo o cumplir con las normativas ambientales, las cuales dificultan la sustentabilidad de la actividad ganadera. Rojas y Vallejo (2016) plantean que las normativas aplicadas a las granjas ganaderas son muy variadas y todas deben ser cumplidas responsablemente. Esto quiere decir que los diferentes sistemas ganaderos tienen un impacto significativo en el ecosistema, por lo que se debe acatar todas las formas de sostenibilidad.

La industria ganadera en América Latina y el Caribe (ALC) se mantiene en un crecimiento continuo y este depende del desarrollo tecnológico. Asimismo, un sinnúmero de factores interviene en el ciclo y desarrollo productivo. Según la Comisión Económica para América Latina y el Caribe (CEPAL, 2017), los componentes fundamentales de la producción de cárnicos en la región son los siguientes: los precios mínimos, el incremento de la producción, el crecimiento per cápita, el cambio de los gustos y preferencias de los clientes en cuanto a las carnes y las nuevas tendencias de producción que minimicen el impacto ambiental.

En Colombia, por ejemplo, se aplicaron diferentes técnicas para la producción de ganado que han mejorado el nivel de productividad. Como manifiestan Pertúz-Martínez y Elías-Caro (2018), la implementación de pasto artificial, el pastoreo y la atención de los animales son parte de los métodos implementados. Estos métodos implican un progreso en el cruce de razas, lo que ayuda a obtener una extracción más limpia tanto de productos lácteos como cárnicos. Estos métodos, además, tienen efecto en diferentes factores económicos, políticos, sociales y sectoriales; un ejemplo claro de ello es el choque entre la demanda y oferta de los productos derivados del ganado (RamosMontaño \& García-Conde, 2016; Mora Marín et al., 2017).

En Ecuador, los sectores agropecuarios son muy importantes debido a que reportan un notable crecimiento dentro de la economía. Morocho et al. (2021) consideran que el sector ganadero requiere de una alta participación de mano de obra para la producción y comercialización. La mano de obra puede ser capacitada en conjunto para la innovación de nuevos materiales útiles para el proceso (Ríos-Núñez \& Benitez-Jiménez, 2015).

Este sector ofrece mayores oportunidades para la población y la economía en conjunto. No obstante, el Ministerio de Agricultura, Ganadería, Acuacultura y Pesca (2016), en cuanto a la política agropecuaria ecuatoriana, dice que esta es una de las 
áreas con mayor vulnerabilidad productiva, por lo que se debe elaborar un análisis que tenga gran relevancia sobre las actividades del mercado agropecuario. Este se convierte en un reto enorme, pero si se lo aborda de manera adecuada, puede generar grandes contribuciones y ayudar a la inclusión económica de las familias ecuatorianas.

El objetivo primordial de este estudio fue establecer una dinámica de mercado para el sector agropecuario de la provincia de $\mathrm{El}$ Oro, por medio del caso Asociación de Ganaderos 24 de mayo. La metodología que se aplicó fue la descriptiva con el fin de establecer la dinámica de mercado como un factor de incidencia en el sector agropecuario-ganadero.

Es importante mencionar que, mediante esta investigación descriptiva, con base en proyecciones hechas en la Asociación de Ganaderos 24 de Mayo (cantón Las Lajas) -la cual produce para un mercado diverso y potencia el agronegocio en la localidad con los siguientes productos: queso, quesillo, carne, leche y ganado vivo- se pudo conocer que la dinámica de mercado del sector ganadero cuenta con un alto consumo de los distintos productos que ofrece.

\section{Revisión de la literatura}

En la economía mundial, el sector agropecuario es estratégico porque oferta alimentos primordiales para la vida y genera fuentes de empleo que aseguran la población de las zonas rurales. Para ejecutarlo, el sector requiere de otras actividades económicas a las cuales se enlaza formando cadenas de valor, por lo que es conocido como el sector creador de la economía (Álvarez, 2020). En suma, esta productividad libera fuerza laboral, lo que incrementa la economía global (Avendaño \& Garza, 2016; Pérez, 2016; Covas-Varela et al., 2017).

En ALC el sector ganadero se caracteriza por dos factores primordiales: la demanda y la producción que son capaces de crear los países. En cuanto a la demanda, esta cuenta con tres tendencias globales: 1) población creciente hasta redondear los 9 mil millones de habitantes hasta el 2050, 2) aumento de los ingresos en su mayoría de la clase mediaalta, y 3) la población que será mayormente urbana (Rizo-Mustelier et al., 2017).

Los productos alimenticios para cualquier país son vitales; como plantea García (2017), en Torreón (México), la producción ganadera es fundamental para que la población sobreviva porque las empresas del sector agropecuario son las unidades económicas que requieren en mayor medida de los recursos naturales con los que cuentan las diferentes regiones, representando la economía interna, la riqueza o, en algunos casos, las carencias del país (Ramírez \& Vallejo, 2016; Nin-Pratt et al., 2019).

En otro ejemplo, en las últimas décadas, el sector agropecuario argentino enfrentó una gran variedad de cambios técnicos, productivos y organizacionales. Torrado y Sili (2020) plantean que esto se debió a las nuevas tecnologías aplicadas y al enfoque en la innovación para generar productos de mayor calidad. El agronegocio produjo un impacto positivo y un crecimiento importante en la producción, y consecuentemente en la comercialización de estos bienes y la relevancia de la participación del capital económico en los sucesos productivos entrelazados con la exportación de mercancía.

De la misma manera, en Ecuador, el sector agropecuario es el mayor generador de divisas y ha tenido una gran importancia después del petróleo. En efecto, Pino et al. (2018) manifiestan que, en la actualidad, el $95 \%$ del consumo se debe a la propia demanda interna de productos agropecuarios y que, además, genera fuentes de empleo para un $25 \%$ de la población económicamente activa. Al analizar el sector agropecuario se puede describir como el único que 
históricamente ha mantenido una economía y balanza comercial positiva, pues sus ventas ganan 9 a 1 en compras. Esto coadyuva a que el Ecuador puede asegurar que goza de soberanía y seguridad alimentaria.

A medida que transcurre el tiempo, explican Castellanos y Solano (2017), las sociedades se desarrollan y con ella evolucionan las tendencias de mercado; tanto es así que, en el contexto de la producción agropecuaria, las empresas, en sus inicios, estaban mayoritariamente influenciadas por la aplicación de procesos manuales con escaso o ningún nivel de automatización, generando productos de escaso valor agregado. Aquel escenario ha cambiado desde hace más de tres décadas en países de notable desarrollo económico.

\section{Dinámica del mercado}

García-Martínez et al. (2015) dice que la dinámica de mercado es considerada dentro de la macroeconomía como un patrón que mueve la relación entre la oferta y la demanda, ya sea de bienes o servicios, para los consumidores. En consecuencia, es fundamental incluirla dentro del plan de negocios de las organizaciones porque brinda resultados sobre las necesidades y preferencias que tienen los clientes dentro de un mercado.

En la dinámica de mercado interviene la oferta, que se refiere a la cantidad que estarían dispuestos a ofrecer en un periodo determinado a un cierto precio. La demanda, de acuerdo con Rodríguez (2017), es representada por el poder de adquisición de los consumidores en un tiempo dado y con un valor, ya sea fijo o cambiante. Estas dos grandes fuerzas hacen que la economía de un país funcione y que los mercados interactúen entre sí. Además, se puede constatar que la dinámica de mercado tiene diversas políticas tanto industriales como gubernamentales.

Hurtado y Sadcidi (2018) plantean que para las organizaciones los efectos de esta dinámica representan quebrantos o lucros. Esto se debe a la interacción entre los ofertantes y los clientes de un mercado, lugar donde interactúan los consumidores. Los consumidores son muy cambiantes debido a que son influenciados por factores, tanto económicos como sociales, y por el contexto nacional e internacional, los cuales afectan la demanda y la oferta. Un ejemplo claro son las barreras arancelarias, las cuales obligan a que la mayor parte de empresas se adapten a sus continuos cambios.

Para este estudio es importante hacer hincapié en los modelos de mercado. Castellanos \& Solano (2017) presentan dos modelos: 1) los de competencia perfecta que se definen como un mercado ideal en donde ni los oferentes ni los demandantes influyen en el precio del producto, sino que este se define de manera libre, y 2), el de competencia imperfecta donde los vendedores y compradores juegan un papel al momento de definir el precio de un producto o servicio. El mercado agropecuario se maneja con una competencia perfecta; es decir, los precios de los productos no son definidos por los consumidores sino por los oferentes quienes, de acuerdo con la dificultad de obtener estos productos, optan por definir un precio adecuado y que genere rentabilidad.

En suma, se puede afirmar que la dinámica del mercado se concentra en la oferta, necesidades y preferencias de los clientes, el precio, cantidad, tiempo determinado de compra, factores del entorno y la demanda.

\section{Materiales y métodos}

Para el correcto desarrollo de la investigación, se utilizó el método descriptivo, a partir de un enfoque cualitativo y cuantitativo, para analizar la dinámica del estudio de mercado del sector agropecuario-ganadero. Es importante decir que Aguirre y Jaramillo (2015, p.175) lo definen como "el estudio 
descriptivo es el método que se puede elegir cuando se deseen descripciones rigurosas de algunos fenómenos. Tal estudio es especialmente útil para investigadores que buscan saber el quién, qué y dónde de los eventos". Asimismo, según Cadena-Iñiguez et al. (2017), es primordial recurrir a fuentes de información tales como revistas científicas para caracterizarlas y analizarlas con el fin de obtener, mediante su narración, información necesaria y relevante.

Al mismo tiempo, el diseño de esta investigación es de tipo no experimental porque no se aplicaron pruebas, sino que se estableció un banco de preguntas con base en los criterios del estudio de mercado. El nivel de la investigación es de tipo explicativo porque se pretendió conocer los factores causales de la actividad ganadera de la asociación, por lo que se consideró a 159 personas como tamaño de la muestra y como objetivo de estudio de la investigación.

Esta exploración fue desarrollada en la Asociación de Ganaderos 24 de mayo, ubicada en la provincia de El Oro, cantón Las Lajas, parroquia La Victoria. Dentro del análisis de involucrados se utilizó la encuesta, que consiste en un cuestionario de preguntas basado en las opiniones vertidas por las 159 personas que forman parte del estudio de mercado de la asociación.

En efecto, la metodología utilizada en el proceso estadístico se describe a través de los documentos de recolección de datos de la dinámica del comportamiento de mercado de la demanda, oferta, precios y canales de distribución de los productos derivados del ganado.

\section{Análisis y resultados}

En la aplicación de los resultados se presenta como evidencia la encuesta del estudio de mercado aplicada a 159 personas. Por medio de esta técnica se pudo identificar los factores clave que inciden en la oferta, demanda, precios y canales de distribución referidos en la asociación. Para este análisis se consideró las tablas de la 1 a la 13 que se muestran a continuación:

\section{Estudio de mercado de la Asociación de Ganaderos 24 de mayo}

Se presenta la encuesta dirigida al mercado mayorista, minorista o consumidores finales de la Asociación de Ganaderos 24 de mayo. El objetivo es investigar el comportamiento del mercado a través de la demanda, oferta, precios y canales de distribución de los productos ganaderos.

La mayor demanda de los productos derivados del ganado (queso, quesillo, carne, leche y ganado vivo) está representada por los comerciantes, con un $34 \%$, quienes compran en mayores cantidades para su posterior venta. Les siguen las amas de casa, las cuales representan el $33.3 \%$, quienes adquieren productos para el consumo dentro del hogar (tabla 1).

De acuerdo con la muestra utilizada, de 159 personas del cantón Las Lajas (parroquia La Victoria) se obtuvo que el $75.5 \%$ son mujeres y el $24.5 \%$, hombres, lo que da un total del $100 \%$. De este resultado se denotó que quienes compran mayor cantidad de productos derivados del ganado (queso, quesillo, carne, leche y ganado vivo) son mujeres casadas -representadas en un $54.10 \%$, en un rango de edad entre 21 y 30 años equivalente al $64 \%$ de total -, debido a que ellas piensan en la alimentación familiar, por ende, siempre buscarán alimento de calidad y, a la vez, económico (tabla 2). 
Tabla 1. Datos generales sobre la ocupación

\begin{tabular}{|l|c|c|}
\hline \multicolumn{1}{|c|}{ Categoría } & Frecuencia & Porcentaje \\
\hline Emprendedor & 4 & $2.5 \%$ \\
\hline Cocinero & 4 & $2.5 \%$ \\
\hline Cocinera & 15 & $9.4 \%$ \\
\hline Ganadero & 5 & $3.1 \%$ \\
\hline Carnicero & 8 & $5.0 \%$ \\
\hline Estudiante & 16 & $10.1 \%$ \\
\hline Comerciante & 54 & $34.0 \%$ \\
\hline Ama de casa & 53 & $33.4 \%$ \\
\hline Total & 159 & $100 \%$ \\
\hline
\end{tabular}

Tabla 2. Datos complementarios

\begin{tabular}{|l|c|c|c|c|c|l|c|c|}
\hline \multicolumn{4}{|c|}{ Género } & \multicolumn{3}{c|}{ Edad } & \multicolumn{3}{c|}{ Estado civil } \\
\hline Categoría & Frec. & $\%$ & Categoría & Frec. & $\%$ & Categoría & Frec. & $\%$ \\
\hline Masculino & 39 & $24.5 \%$ & $18-20$ & 10 & $6 \%$ & Casado & 14 & $8.80 \%$ \\
\hline Femenino & 120 & $75.5 \%$ & $21-30$ & 101 & $64 \%$ & Casada & 86 & $54.10 \%$ \\
\hline Total & 159 & $100 \%$ & $31-40$ & 42 & $28 \%$ & Unión libre & 2 & $1.30 \%$ \\
\hline & & & $41-50$ & 4 & $2 \%$ & Soltero & 24 & $15.10 \%$ \\
\hline & & & Total & 159 & $100 \%$ & Soltera & 33 & $20.70 \%$ \\
\hline & & & & & & Total & 159 & $100 \%$ \\
\hline
\end{tabular}

Pregunta 1. ¿Cuál es el producto derivado del ganado que usted más adquiere y con qué frecuencia lo hace?

Los productos derivados del ganado (queso, quesillo, carne, leche y ganado vivo) más consumidos por la población de La Victoria es la leche, con un $39 \%$, considerada un producto que se puede ofertar en mayor cantidad para satisfacer a toda la demanda. A este le siguen carne, queso y quesillo. El producto menos adquirido por la población es el ganado vivo, con un $3.1 \%$. En este último se deberían emplear recursos y estrategias para incrementar su reconocimiento y demanda (tabla 3).

Tabla 3. Producto ganadero que más adquiere

\begin{tabular}{|c|c|c|c|c|c|c|}
\hline \multirow{2}{*}{ Categoría } & \multicolumn{4}{|c|}{ Adquisición } & \multirow{2}{*}{ Frec. } & \multirow{2}{*}{$\%$} \\
\hline & Diario & Semanal & Quincenal & Mensual & & \\
\hline Quesillo & 105 & 41 & 10 & 3 & 19 & $11.9 \%$ \\
\hline Leche & 100 & 51 & 6 & 2 & 62 & $39 \%$ \\
\hline Carne & 30 & 97 & 24 & 8 & 36 & $22.6 \%$ \\
\hline Queso & 106 & 46 & 5 & 2 & 37 & $23.3 \%$ \\
\hline Ganado vivo & 1 & 11 & 7 & 140 & 5 & $3.2 \%$ \\
\hline Total & & & & & 159 & $100 \%$ \\
\hline
\end{tabular}


Pregunta 2. ¿Qué tan probable es que recomiende o reemplace el producto derivado del ganado con otro? (Queso, quesillo, leche, carne y ganado vivo).

En la población de La Victoria, los consumidores de los productos derivados del ganado manifestaron que es probable que cambien su producto actual, independientemente de las razones que sean, o que tiendan a recomendar a otros consumidores los productos que adquieren. Por este motivo, es fundamental que la asociación esté en constante innovación, para satisfacer y mantener la fidelidad de los clientes y ampliar su mercado objetivo (tabla 4).

Tabla 4. Probabilidad de reemplazar o recomendar el producto ganadero

\begin{tabular}{|l|c|c|}
\hline \multicolumn{1}{|c|}{ Categoría } & Frecuencia & Porcentaje \\
\hline Muy Probable & 19 & $3.2 \%$ \\
\hline Probable & 83 & $52.2 \%$ \\
\hline Poco probable & 53 & $33.3 \%$ \\
\hline Nada probable & 18 & $11.3 \%$ \\
\hline Total & 159 & $100 \%$ \\
\hline
\end{tabular}

Pregunta 3. ¿Cuáles son las características que usted observa para adquirir el producto?

Según la encuesta, la población de La Victoria, para adquirir productos ganade- ros (queso, quesillo, leche, carne y ganado vivo), se basa, especialmente, en la calidad del producto, luego en el precio y un mínimo porcentaje de la población se fija en el peso (tabla 5).

Tabla 5. Características del producto ganadero

\begin{tabular}{|l|c|c|c|c|c|}
\hline \multicolumn{1}{|c|}{ Categoría } & Saludable & Calidad & Precio & Peso & Total \\
\hline Quesillo & 9 & 109 & 37 & 4 & 159 \\
\hline Leche & 15 & 116 & 26 & 2 & 159 \\
\hline Carne & 23 & 113 & 12 & 11 & 159 \\
\hline Queso & 11 & 111 & 35 & 2 & 159 \\
\hline Ganado vivo & 13 & 124 & 13 & 9 & 159 \\
\hline
\end{tabular}

Pregunta 4. ¿Califique el nivel de satisfacción y atención al momento de realizar la compra del producto ganadero? (Queso, quesillo, leche, carne y ganado vivo).

La satisfacción que muestran los clientes al adquirir los derivados del ganado (queso, quesillo, carne, leche y ganado vivo) es buena; sin embargo, esta se podría mejorar si la asociación toma en cuenta las necesidades de los consumidores, además de aplicar innovaciones en la organización, por ejemplo, capacitaciones sobre atención al cliente para la mejora continua de la asociación (tabla 6).

Tabla 6. Nivel de satisfacción

\begin{tabular}{|l|l|l|}
\hline \multicolumn{1}{|c|}{ Categoría } & \multicolumn{1}{c|}{ Frecuencia } & \multicolumn{1}{c|}{ Porcentaje } \\
\hline Muy satisfactorio & 33 & $20.8 \%$ \\
\hline Satisfactorio & 120 & $75.5 \%$ \\
\hline Poco satisfactorio & 6 & $3.7 \%$ \\
\hline Nada satisfactorio & 0 & $0 \%$ \\
\hline Total & 159 & $100 \%$ \\
\hline
\end{tabular}


Pregunta 5. Cuándo hace un pedido de productos ganaderos (queso, quesillo, leche, carne y ganado vivo), ¿el tiempo de respuesta es?
Los consumidores expresaron que al momento de hacer un pedido lo adquieren de manera inmediata; es decir, la transacción de compra y venta es instantánea, por ende, es un servicio de calidad (tabla 7).

Tabla 7. Tiempo de respuesta para hacer un pedido

\begin{tabular}{|l|c|c|}
\hline \multicolumn{1}{|c|}{ Categoría } & Frecuencia & Porcentaje \\
\hline Inmediato & 143 & $89.9 \%$ \\
\hline De 1 a 3 meses & 11 & $6.9 \%$ \\
\hline De 3 a 6 meses & 3 & $1.9 \%$ \\
\hline De 6 meses en adelante & 2 & $1.3 \%$ \\
\hline Total & 159 & $100 \%$ \\
\hline
\end{tabular}

Pregunta 6. ¿Considera que los vendedores generan cambios innovadores en los diferentes productos ganaderos de los cuales usted se ha beneficiado?

La mayor parte de los clientes desconoce los cambios innovadores aplicados en los productos ganaderos de la asociación. Es necesario implementar nuevos recursos de promoción y publicidad para dar a conocer la cartera de bienes con la información necesaria: el precio, características, beneficios, entre otros (tabla 8).

Tabla 8. Cambios innovadores en los productos

\begin{tabular}{|l|c|c|}
\hline \multicolumn{1}{|c|}{ Categoría } & Frecuencia & Porcentaje \\
\hline Sí & 40 & $25.2 \%$ \\
\hline No & 32 & $20.1 \%$ \\
\hline Desconozco & 87 & $54.7 \%$ \\
\hline Total & 159 & $100 \%$ \\
\hline
\end{tabular}

Pregunta 7. ¿Cómo calificaría la relación calidad precio del producto ganadero?

Las 159 personas encuestadas consideran entre buena y muy buena la relación entre la calidad y el precio del producto, lo que supone un aspecto positivo para los productos ofertados por la asociación (tabla 9).

Tabla 9. Relación calidad y precio del producto ganadero

\begin{tabular}{|l|c|c|}
\hline \multicolumn{1}{|c|}{ Categoría } & Frecuencia & Porcentaje \\
\hline Bueno & 74 & $46.50 \%$ \\
\hline Muy bueno & 63 & $39.60 \%$ \\
\hline Regular & 22 & $13.8 \%$ \\
\hline Malo & 0 & $0 \%$ \\
\hline Total & 159 & $100 \%$ \\
\hline
\end{tabular}


Pregunta 8. ¿Indique a qué asociación, empresa o persona natural del sector usted le compra los productos ganaderos? (Queso, quesillo, leche, carne y ganado vivo).

Los consumidores se inclinan a hacer sus compras en la Asociación de Ganaderos
24 de mayo. La asociación oferta una variedad de productos de buena calidad, lo que la hace que sea reconocida localmente. A esta le siguen otras asociaciones que brindan productos similares (tabla 10).

Tabla 10. Asociación, empresa o persona natural

\begin{tabular}{|l|c|c|}
\hline \multicolumn{1}{|c|}{ Categoría } & Frecuencia & Porcentaje \\
\hline Coop. de Producción Ganadera La Victoria & 58 & $56 \%$ \\
\hline Coop. de Producción Ganadera 24 de Mayo & 89 & $36.5 \%$ \\
\hline ECOLAC & 7 & $4.4 \%$ \\
\hline Mercado & 5 & $3.1 \%$ \\
\hline Total & 159 & $100 \%$ \\
\hline
\end{tabular}

Pregunta 9. ¿Cuál fue el medio de comunicación que le hizo conocer la oferta de los productos ganaderos de la Asociación de Ganaderos 24 de mayo? (Queso, quesillo, leche, carne y ganado vivo).

La mayoría de los consumidores conoce los productos de la asociación mediante las redes sociales. Si bien es cierto que actualmente muchas personas de diferentes rangos de edad utilizan la tecnología, hay clientes que conocen más de los productos por medio de anuncios en televisión y radio (tabla 11).

Tabla ll. Medio de comunicación que le hizo conocer la oferta de los productos ganaderos

\begin{tabular}{|l|c|c|}
\hline \multicolumn{1}{|c|}{ Categoría } & Frecuencia & Porcentaje \\
\hline Radio & 20 & $12.6 \%$ \\
\hline Televisión & 32 & $20.1 \%$ \\
\hline Reuniones & 19 & $11.9 \%$ \\
\hline Redes sociales & 88 & $55.3 \%$ \\
\hline Total & 159 & $100 \%$ \\
\hline
\end{tabular}

Pregunta 10. Indique qué tipo de comprador es usted en relación con los productos ganaderos.

La mayor demanda de productos ganaderos está conformada por los consumidores finales, es decir, que quienes más compran son las familias, las cuales buscan productos de calidad $y$, a su vez, abaratar costos comprando de forma directa. Después figuran los compradores mayoristas y minoristas, quienes buscan productos de primera para satisfacer la demanda de sus establecimientos (tabla 12). 
Tabla 12. Tipo de comprador en relación a los productos ganaderos

\begin{tabular}{|l|c|c|}
\hline \multicolumn{1}{|c|}{ Categoría } & Frecuencia & Porcentaje \\
\hline Mayorista & 21 & $13.2 \%$ \\
\hline Minorista & 64 & $40.3 \%$ \\
\hline Consumidor final & 74 & $46.5 \%$ \\
\hline Total & 159 & $100 \%$ \\
\hline
\end{tabular}

Las personas que más consumen los productos derivados del ganado son las amas de casa que buscan la calidad y un precio al alcance de su economía. Asimismo, se denotó que el producto más consumido es el queso seguido de la leche.
Pregunta 11. ¿Dónde le gustaría adquirir el producto ganadero?

De las 159 personas encuestadas, en su mayoría, les gustaría adquirir sus productos en los mercados al igual que en tiendas cercanas a su domicilio (tabla 13).

Tabla 13. ¿Dónde le gustaría adquirir el producto ganadero?

\begin{tabular}{|l|c|c|}
\hline \multicolumn{1}{|c|}{ Categoría } & Frecuencia & Porcentaje \\
\hline Tiendas físicas & 41 & $25.8 \%$ \\
\hline Tiendas en línea & 10 & $6.3 \%$ \\
\hline Tercenas & 35 & $22 \%$ \\
\hline Mercados & 62 & $39 \%$ \\
\hline Supermercados & 11 & $6.9 \%$ \\
\hline Total & 159 & $100 \%$ \\
\hline
\end{tabular}

\section{Discusión y conclusiones}

A continuación, se presenta la proyección en la cual se expone el faltante de productos, de forma porcentual, para que la asociación mantenga el mercado abastecido y brinde mejores servicios dentro de los cinco años venideros.

Tabla 14. Proyección de la demanda insatisfecha

\begin{tabular}{|l|c|c|c|c|c|}
\hline \multirow{2}{*}{\begin{tabular}{c}
\multirow{2}{*}{$\begin{array}{c}\text { Productos } \\
\text { derivados }\end{array}$} \\
\cline { 2 - 6 }
\end{tabular}} & $\mathbf{1}$ & $\mathbf{2}$ & $\mathbf{3}$ & $\mathbf{4}$ & $\mathbf{5}$ \\
\hline Leche & $2.33 \%$ & $2.42 \%$ & $2.51 \%$ & $2.60 \%$ & $2.69 \%$ \\
\hline Queso & $42.81 \%$ & $44.45 \%$ & $46.09 \%$ & $47.72 \%$ & $49.36 \%$ \\
\hline Quesillo & $4.95 \%$ & $5.07 \%$ & $5.20 \%$ & $5.32 \%$ & $5.44 \%$ \\
\hline Carne & $15.71 \%$ & $15.80 \%$ & $15.89 \%$ & $15.97 \%$ & $16.06 \%$ \\
\hline Ganado vivo & $2.62 \%$ & $2.66 \%$ & $2.69 \%$ & $2.73 \%$ & $2.77 \%$ \\
\hline
\end{tabular}

Dentro de este estudio se hizo una proyección general de la demanda de los productos derivados del ganado utilizando datos de los últimos cinco años de la Asociación de Ganaderos 24 de mayo, además de la tasa de crecimiento poblacional. Estos datos en con- 
junto con la encuesta arrojaron como resultado que existe una demanda insatisfecha.

Una demanda insatisfecha, de acuerdo con la interpretación de Fuentes et al. (2017), es el descontento que tienen los consumidores con las necesidades expresadas en un mercado ofertante. En este contexto, Rizo-Mustelier et al., (2017), en su teoría, infieren que es recomendable que los comerciantes mantengan una producción más activa $y$, de esta forma, logren obtener un equilibrio entre oferta y demanda sin que se incrementen los costos de manufactura. Esto es posible mediante estrategias que, por un lado, ayuden a fidelizar a los consumidores y, por el otro, que ellos no opten por consumir productos sustitutos.

Una vez determinados los flujos de la Asociación de Ganaderos 24 de Mayo (cantón las Lajas), se puede deducir que sí existen dichas insatisfacciones dentro del rango proyectado en relación con los productos derivados del ganado, ya que hay una diferencia clara en la proyección de la demanda insatisfecha. Por tal motivo, se anima a la asociación a que comercialice los productos en mayores cantidades, principalmente el queso porque la proyección en el año uno es de $42.81 \%$ y se incrementa anualmente con el $2 \%$ hasta el año cinco en $49.36 \%$ de la demanda insatisfecha. El queso es el producto de mayor consumo entre los clientes dentro de los años proyectados.

En el estudio se constató que los consumidores, ya sean minoristas o mayoristas, no logran captar la información sobre la variedad de productos que oferta la asociación, por ende, esta tiende a ser muy escasa al momento de hacer publicidad y promociones. Consecuentemente, se destacaron las características que mayor influencia tienen en los consumidores al momento de adquirir un producto haciendo referencias porcentuales. El $54 \%$ de los consumidores está representado por mujeres, quienes hacen observaciones al momento de la compra, ya sea en calidad o precio, destacando así los productos con mayor demanda, donde figuran la leche con el $39 \%$ y el queso con el $23.3 \%$ del total.

Aparte, si bien la Asociación de Ganaderos 24 de Mayo ofrece una buena calidad en los productos y excelente atención al cliente, necesita aumentar su capacidad tanto en recursos materiales como en capital humano para solventar la demanda insatisfecha, la cual está en constante aumento. Con base en la proyección calculada para los siguientes cinco años, la asociación tendrá una insatisfacción anual del $2 \%$, lo cual se relaciona con el incremento o decrecimiento de los ingresos generados por los productos ganaderos.

En última instancia, se recomienda impulsar a los productores ganaderos a que apliquen nuevos métodos tanto para la producción como para la comercialización de sus productos, para generar una dinámica de mercado más efectiva, relacionada con el incremento de la oferta y la demanda de la asociación, principal generadora de fuentes de empleo de la localidad.

\section{Referencias}

Aguirre, J., \& Jaramillo, L. (2015). El papel de la descripción en la investigación cualitativa. Cinta de Moebio (53), 175-189. http://dx.doi.org/10.4067/S0717554X2015000200006

Álvarez, A. (2020). El sector agropecuario y el desarrollo económico: El caso cubano. Economía y Desarrollo, 164(2), 1-23. https:// bit.ly/3B3rUR7

Avendaño, V., \& Garza, P. (2016). Estructura de razas, cruzas y criollos de ganado bovino en los sistemas productivos de la región Tulijá-Tseltal-Chol en Chiapas, México. Archivos de Zootecnia, 65(249), 35-42. https://bit.ly/3B6wY7p

Cadena-Iñiguez, P., Rendón-Medel, R., AguilarÁvila, J., Salinas-Cruz, E., De la Cruz, F., 
\& Sangerman-Jarquin. (2017). Métodos cuantitativos, métodos cualitativos o su combinación en la investigación: Un acercamiento en las ciencias sociales. Revista Mexicana de Ciencias Agrícolas, 8(7) 1603-1617. https://bit.ly/3juGb3y

Castellanos, G., \& Solano, D. (2017). Metaanálisis de la relación entre la orientación al mercado y los resultados de la empresa. Estudios Gerenciales, 33(142), 87-94. https:// doi.org/10.1016/j.estger.2016.10.006

Comisión Económica para América Latina y el Carible (CEPAL). (2017). Perspectivas de la agricultura y del desarrollo rural en las Américas: Una mirada hacia América Latina y el Caribe 2017-2018. CEPAL; FAO; IICA. https://bit.ly/3Gqqm7Z

Covas-Varela, D., Martínez-Curbelo, G., DelgadoÁlvarez, N., \& Díaz-Peña, M. (2017). Mejora de procesos logísticos en la comercializadora Cienfuegos. Ingeniería Industrial, 38(2), 210-222. https://bit.ly/3C8y0G5

Fuentes, L., Link, F. \& Valenzuela, F. (2017). Impactos de la dinámica urbana en los mercados laborales en las principales ciudades. Cadernos Metropole, 19(38), 157-177. https://bit.ly/3juNOC7

García-Martínez, A., Abarrán-Portillo, B., \& Avilés, F. (2015). Dinámicas y tendencias de la ganadería doble propósito en el sur del Estado de México. Agrociencia, 49(2), 125-139. https://bit.ly/3B8b6bQ

García, S. (2017). Las empresas agropecuarias y la administración financiera. Revista Mexicana de Agronegocios, 40(ene.-jun.), 583-594. https://bit.ly/3Ge30XQ

Hurtado, A. J., \& Sadcidi, Z. (2018). Reflexiones acerca de los efectos estáticos y dinámicos del mercado común del sur 1. Aldea Mundo, 23(46), 21-30. https://bit. ly/2ZfFpzU

Ministerio de Agricultura, Ganadería, Acuacultura y Pesca. (2016). La política agropecuaria ecuatoriana: Hacia el desarrollo territorial rural sostenible 2015-2025 (Parte I). https://bit.ly/3E9arzD

Mora Marín, M.A, Ríos Pescador, L., Ríos Ramos, L., \& Almario Charry, J. L. (2017). Impacto de la actividad ganadera sobre el suelo en
Colombia. Ingeniería y Región, 17, 1-12. doi: https://doi.org/10.25054/22161325.1212

Morocho, B., Carvajal, H., \& Vite, H. (2021). Análisis socioeconómico del agronegocio ganadero: Caso agro productores de la Aso Ganaderos del Altiplano Orense 5 de noviembre del cantón Atahualpa. Revista Metropolitana de Ciencias Aplicadas, 4(1) 26-32. https://bit.ly/3b48aCt

Nin-Pratt, A., Freiria, H., \& Muñoz, G. (2019). Productividad y eficiencia en la producción ganadera pastoril en América Latina. Banco Interamericano de Desarrollo. https:// bit.ly/3no5506

Pérez, L. (2016). La ganadería bovina en el nororiente de Colombia: Fomento de razas y dinámicas en la propiedad de tierras en Socorro, XVIII. Cuadernos de Economía, 35(68), 521-545. https://bit.ly/3c8e7dq

Pertúz-Martínez, A. P., \& Elías-Caro, J. E. (2018). Riesgo y poder en las organizaciones ganaderas en Colombia: Enfoque desde la historiografía económica, social y empresarial. Clío América, 12(24), 202-218. https://doi.org/10.21676/23897848.3014

Pino, S., Aguilar, H., Azuero, E., \& Sisalema, L. (2018). Aporte del sector agropecuario a la economía del Ecuador: Análisis crítico de su evolución en el período de dolarización. Años 2000-2016. Espacios, 39(32), 7. https://bit.ly/3EcNNzk

Ramírez, J., \& Vallejo, R. (2016). Las actividades ganaderas en Jalisco, México: Cumplimiento ambiental de tratamiento de residuos sólidos y líquidos presentado por el sector productivo ante las instituciones ambientales. Revista Mexicana Agropecuaria, 39(jul.-dic.), 423-440. https://bit. ly/3B9pqk8

Ramos-Montaño, C., \& García-Conde, M. (2016). Características ecosistémicas asociadas a la actividad ganadera en Arauca (Colombia): Desafíos frente al cambio climático. Orinoquia, 20(1), 28-38. https://bit. ly/300UVnf

Ríos-Núñez, \& Benitez-Jiménez. (2015). Análisis del funcionamiento económico-productivo de los sistemas de producción cárnica bovina en la Amazonía ecuatoriana. Ar- 
chivos de Zootecnia, 64(248), 409-416. https://doi.org/10.21071/az.v64i248.428

Rizo-Mustelier, M., Villa-Tabares, B., Vuelta-Lorenzo, D., \& Vargas-Batis, B. (2017). Estrategias de comercialización para la gestión de ventas en el mercado agropecuario estatal ferreiro de Santiago de Cuba. Ciencia en su PC (4), 91-102. https://bit. ly/3E5X0JZ

Rojas, J., \& Vallejo, R. (2016). Las actividades ganaderas en jalisco, méxico: cumplimiento ambiental de tratamiento de residuos sólidos y líquidos presentado por el sector productivo ante las instituciones ambientales. Revista Mexicana de Agrone- gocios, 39(jul.-dic.), 423-440. https://bit. ly/3BcD2v8

Torrado, R., \& Sili, M. (2020). Toma de decisiones y gestión productiva en el sector agropecuario del Noreste de La Pampa (Argentina). Economia e Sociologia Rural, 58(2), 1-19. https://doi.org/10.1590/18069479.2020.198357

Verdoljak, J., Pereira, M., Gándara, L., Acosta, F., Fernández-López, C., \& Martínez-González, J. (2018). Reproducción y mortalidad de razas bovinas en clima subtropical de Argentina. Abanico Veterinario, 8(1) 28-35. https://bit.ly/2XDnvXw 